SESSION VII. EVOLUTION - Chair: Peter S. Conti 


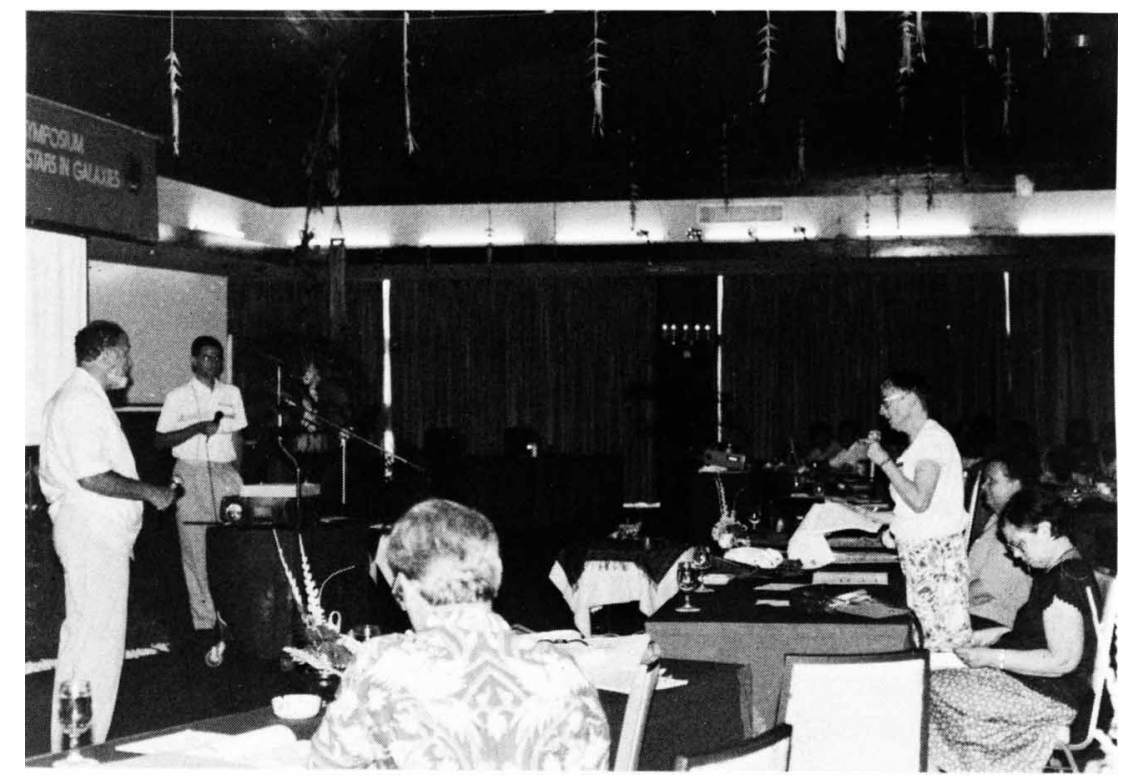

Conti chairing, Leitherer, Underhill, Niemela, Lozinskaya

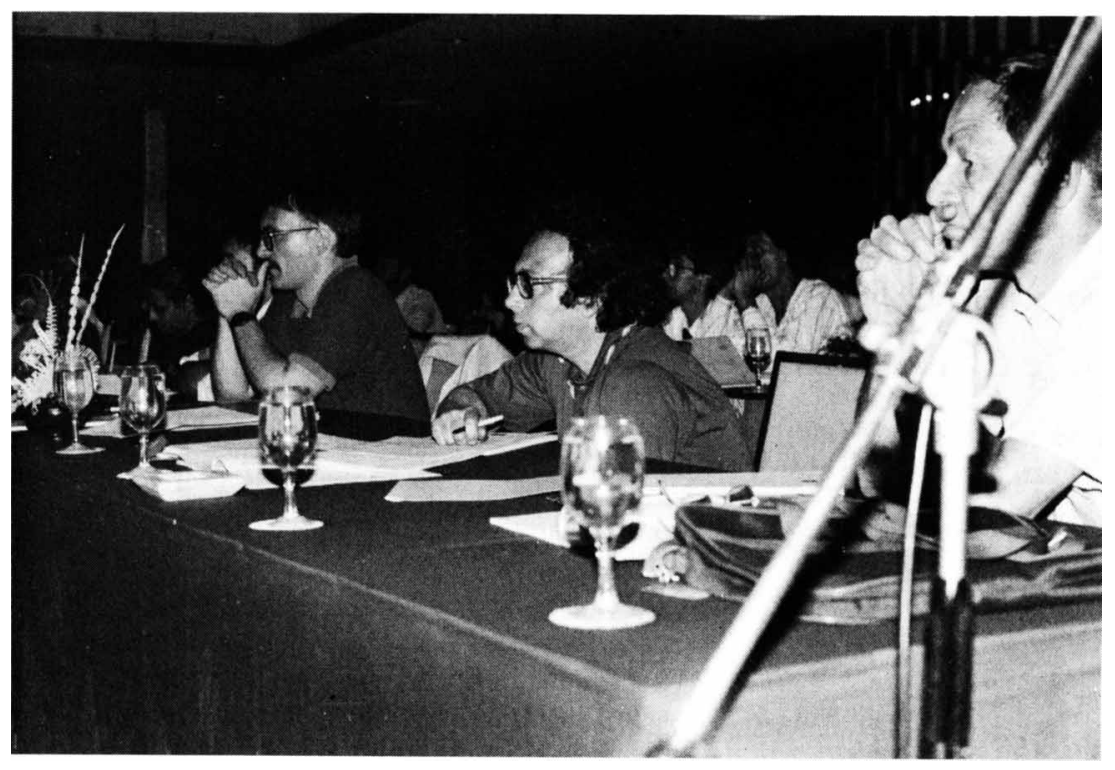

Andy Pollock, Lev Yungelson, Arnout van Genderen 


\title{
STELLAR EVOLUTION IN THE UPPER HRD: THEORY
}

\author{
NORBERT LANGER \\ Universitäts-Sternwarte Göttingen \\ Geismarlandstrasse 11, D-3400 Göttingen, F.R.G.
}

\begin{abstract}
Theoretical aspects of the modeling of observable evolutionary phases of massive single stars are reviewed. The SN 1987A progenitor evolution is considered in detail as an example for a star below the WR limit. Formation, structure, evolution, and mass loss of WR stars are discussed, and the impact of supernova research on stellar evolution theory is stressed.
\end{abstract}

\section{Introduction}

It is the aim of this review to outline both, recent progress and actual problems of the theory of massive star evolution. Due to space limitations, this work will be far from comprehensive. Instead it will focus on a rather subjective selection of topics with - of course - some emphasis on Wolf-Rayet (WR) stars. This paper concentrates on theoretical aspects of structure and evolution of massive stars. Some effects can be understood easily within a high degree of simplification and abstraction. Therefore, it is sometimes argued in a rather schematic way, e.g. on the basis of schematic diagrams instead of results of actual calculations. It should be noted though, that all conclusions have been verified by detailed model calculations. Finally, the comparison of theoretical stellar evolution results with observations of massive stars deserves more space than it could actually be given here for briefty reasons; the reader is refered to the quoted original literature for that purpose.

The topic of this paper is restricted to observable phases of non-rotating, nonmagnetic, massive $\left(M_{Z A M S} \gtrsim 15 M_{\odot}\right)$ single stars (and even for this it cannot be complete). For the non-specialist it may be surprising, that despite such simplifications already the modelling of the earliest evolutionary stages of massive stars are complicated by many problems, as shown in Sect. 2. Relatively much space is devoted to the progenitor evolution of SN 1987A, which is a challenge to stellar evolution theory due to the multitude of tight observational constraints. Sections 3 and 4 deal with the theory of the formation and evolution of WR stars, respectively, while Section 5 briefly outlines the impact of supernova studies on the theory of massive star evolution. 


\section{Early evolutionary phases}

Already the modeling of the main sequence phase of massive stars encounters two main problems of stellar evolution theory, namely mass loss and convection. Possibly, massive stars lose a significant fraction of their initial mass on the main sequence, which potentially affects structure and evolution on the main sequence and beyond (cf. Chiosi and Maeder, 1986). Further, massive main sequence stars are very hot, not only at their surface but throughout their interior, as compared to lower mass stars. Radiation pressure is important, which makes convection more likely to occur. Consequently, besides large convective cores, also parts of the envelopes of massive main sequence stars may obtain a superadiabatic temperature stratification, which in connection with mean molecular weigth gradients, leads to the problem of semiconvection (see below). Also the extension of the convective core itself has to be regarded as uncertain due to the unknown efficiency of convective overshooting (cf. Langer, 1986; Renzini, 1987).

a) Main sequence mass loss

In recent years, a quantitative mass loss theory applicable to massive main sequence stars has been developed (Castor et al., 1975; Abbott, 1982; Pauldrach et al., 1986; Owocki et al., 1988), which in principle allows the calculation of the mass loss rate for a given stellar model. However, due to the large numerical effort involved in stellar wind models, completely selfconsistent coupled stellar wind-stellar evolution calculations are not yet available. A step in this direction has recently been done by Langer and El Eid (1990), who performed stellar evolution calculations using the analytical wind solutions of Kudritzki et al. (1989), which approximate hydrodynamic wind models to high precision. The result is - at galactic metallicity a decrease of the total amount of mass lost during main sequence evolution by a factor $2-3$ as compared with calculations using empirical mass loss rates. Leitherer and Langer (1990), who calculated selfconsistent ZAMS star-wind models for various metallicities $Z$ and found $\dot{M} \sim Z^{0.6 \ldots 0.7}$, estimate main sequence mass loss to be negligible (i.e. $\Delta M / M<5 \%$ for main sequence evolution) for stars less massive than $32 M_{\odot}$ at solar metallicity, and $50 M_{\odot}$ and $80 M_{\odot}$ for LMC and SMC, respectively. Thus, according to current theoretical mass loss rates, main sequence mass loss may have been considerably overestimated in recent years.

The reason for the discrepancy of theoretical and empirical mass loss rates on one side but the good agreement of theoretical wind models when compared in detail with observations of individual stars needs further investigation (cf. Pauldrach et al., 1990). For a discussion of evolutionary consequences cf. Chiosi and Maeder (1986), Langer (1990).

b) The role of semiconvection for stars below the Humphreys-Davidson limit

The expression "semiconvection" has different meanings for different astronomers. Here, it is used for the vibrational instability found by Kato (1966) for superadiabatic layers (i.e. $\nabla>\nabla_{a d}$ ) which contain a positive gradient of the mean molecular weight $\mu$ (i.e. $\nabla_{\mu}:=\frac{d \ln \mu}{d \ln P}>0$ ). More specifically, this instability occurs for $\nabla_{a d}<\nabla<\nabla_{a d}+\delta \nabla_{\mu}=: \nabla_{L}$, where $\nabla_{a d}=\left(\frac{\partial \ln T}{\partial \ln P}\right)_{a d}, \quad \nabla=\frac{d \ln T}{d \ln P}$, and $\delta=-\left(\frac{\partial \ln \rho}{\partial \ln T}\right)_{P}$, i.e. for a situation where the Schwarzschild criterion indicates instability $\left(\nabla_{a d}<\nabla\right)$ and the Ledoux criterion stability $\left(\nabla<\nabla_{L}\right)$. Kato also showed, that the condition for the onset of convection is the Ledoux criterion 
(Ledoux, 1941). On the basis of Kato's analysis, Langer et al. (1983) calculated timescale and diffusion coefficient $D_{s c}$ of mixing due to semiconvection as

$$
D_{s c}=\frac{\alpha}{6} \frac{\nabla-\nabla_{a d}}{\nabla_{L}-\nabla} D_{r a d}
$$

with the radiation diffusion coefficient $D_{r a d}=\frac{\sigma}{c_{P} \rho}, \sigma=\frac{4 a c}{3 \kappa \rho} T^{3} . \alpha$ is an efficiency parameter of order 0.1 (Langer et al., 1985).

This understanding of semiconvection is similar to that of Weaver et al. (1978) as discussed in Langer et al. (1990), and recovers what has been called "semiconvective neutrality" (i.e. $\nabla \equiv \nabla_{a d}$, cf. Chiosi and Summa, 1970; Eggleton, 1972; Stothers and Chin, 1976; Iben, 1974) in the case of $\tau_{s c} \ll \tau_{e v}$, i.e. when the evolutionary timescale is large compared to the semiconvective mixing time. Note that $\tau_{s c} \ll \tau_{e v}$ is only valid for semiconvective zones in the envelopes of massive main sequence stars. In this stage, semiconvection is only of little importance (cf. Chiosi and Nasi, 1978). However, semiconvection during H-shell burning (i.e. the contraction phase towards core helium ignition) largely affects the H-profile and thereby the surface temperature evolution (i.e. the HRD track) during core Heburning (Lauterborn et al., 1971ab; Kozlowski, 1971), and semiconvection above the convective He-burning core controls the final mass of the $\mathrm{C} / \mathrm{O}$-core and possible blue loops after core He-depletion (Langer et al., 1989; see below). In both cases it is $\tau_{s c} \simeq \tau_{e v}$, which makes a timedependent treatment of semiconvective mixing inevitable.

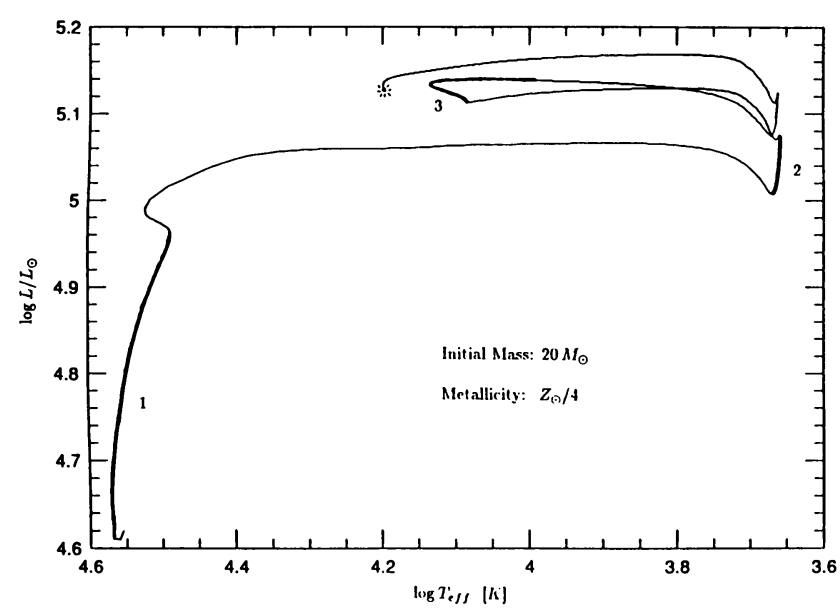

Fig. 1: Theoretical evolutionary track of a $20 M_{\odot}$ star of LMC composition computed with semiconvection (see text) as model for the presupernova evolution of the SN 1987A progenitor. Slow evolutionary phases $(1=$ main sequnece, $2=\mathrm{RSG}, 3=\mathrm{BSG}$ ) are indicated by the thick drawn parts of the track. effects

An example for the of semiconvection, which has recently been studied in Göttingen in great detail, is the progenitor evolution of supernova $(\mathrm{SN})$ 1987A. Stellar evolution sequences for a $20 M_{\odot}$ star of LMC composition have been computed using Eq. (1), for different values of the semiconvective efficiency parameter $\alpha$. All other physical ingredients were up-to-date but standard (cf. Langer et al., 1989, for details). The sequence which can at best account for the observational constraints for the SN 1987A progenitor evolution (cf. Arnett et al., 1989) has been obtained with $\alpha=0.04$

(which is not too far from our order of magnitude estimate of $\alpha \simeq 0.1$; cf. Langer et al., 1985). The corresponding evolutionary track is displayed in Fig. 1; it is strongly 
influenced by semiconvection in the following way.

Due to the reduced efficiency of mixing in the presence of $\mu$-gradients as compared to calculations which use the Schwarzschild criterion for convection, the intermediate convection zone which develops during $\mathrm{H}$-shell burning is limited in extension. In particular, the homogenization of the intermediate layers does not extend downwards up to the location of the hydrogen burning shell but only up to a mass fraction $q_{0}$. Therefore, the H-burning shell is confined to a region of low hydrogen concentration during early phases of core helium burning, i.e. the shell generates a relatively small amount of luminosity. This is known to lead to a red supergiant (RSG) structure (cf. Stothers and Chin, 1976; Langer et al., 1985) and explains the rapid evolution of our model to the Hayashi line after core H-exhaustion.

During core helium burning the H-burning shell moves outwards and eventually reaches the location $q_{0}$, where suddenly the hydrogen concentration becomes high. This leads to an activation of the $\mathrm{H}$-burning shell (the generated luminosity due to $\mathrm{H}$-shell burning almost doubles within a thermal timescale) and consequently the star moves to the blue supergiant (BSG) region in the HR diagram (cf. Lauterborn et al., 1971ab; Fricke and Strittmatter, 1972), where it remains until core Heexhaustion, which leads the star back to the Hayashi-line. This is how the hydrogen profile determines the evolutionary track during core He-burning.

Beyond core-He exhaustion a He-burning shell

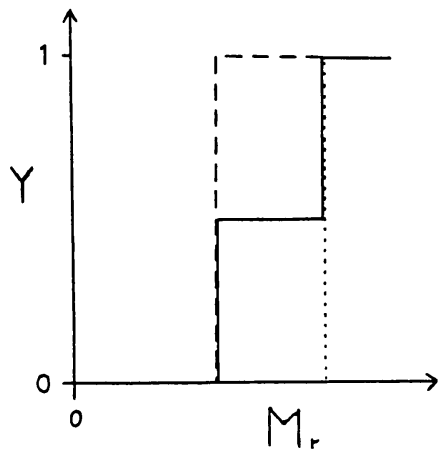

Fig. 2: Schematic He-profiles at time

core He-exhaustion for three different assumptions on convection, i.e. the Ledoux criterion (dashed line), the Schwarzschild criterion (dottet line), and semiconvection (solid line). is activated, and consequently the He-profile becomes important. Fig. 2 shows schematically the He-profiles at the time of core He-exhaustion for three assumptions on convection, i.e. according to calculations performed with the Schwarzschild criterion for convection and with the Ledoux criterion for convection, respectively, as well as the case with semiconvection (as defined above). Only the latter case results in a low concentration of helium at the location of the He-burning shell, which is due to the slow semiconvective mixing of helium inside and He-burning products outside the convective core during central helium burning. For the Schwarzschild- or the Ledoux criterion, $Y \simeq 1$ is the result. The He-concentration at the location of the He-burning shell may be particularly important since He-burning due to the $3 \alpha$-reaction depends on the cube of the He-concentration.

In order to understand the final blue loop of our sequence (cf. Fig. 1) we have to invoke the so called "mirror principle", which is an empirical law stating that an actively burning shell turns a contraction below it into an expansion above it and vice versa (cf. Kippenhahn and Weigert, 1990). Beyond core He-exhaustion the He-burning shell is certainly active and transforms the core contraction towards carbon burning into an expansion of the layers above it. Usually, this expansion is sufficiently strong in order to quickly reduce the temperature at the location of the H-burning shell, which consequently fades away and thus cannot influence the radial motions of the envelope any more. In this case the whole envelope is expanding. In case of semiconvection, however, the activity of the He-burning shell is reduced due to the low concentration of fuel (Fig. 2), which means that the expansion of the overlying layers is less strong as 
compared with the cases of Schwarzschild or Ledoux criterion. As a result the Hburning shell remains active during the whole contraction phase towards C-ignition and changes - according to the mirror principle - the expansion below into an envelope contraction above (see Fig. 3). This is the reason why the star turns into a BSG after core He-exhaustion in the case of semiconvection, but remains in the RSG stage if just the Schwarzschild- or the Ledoux criterion for convection is invoked.
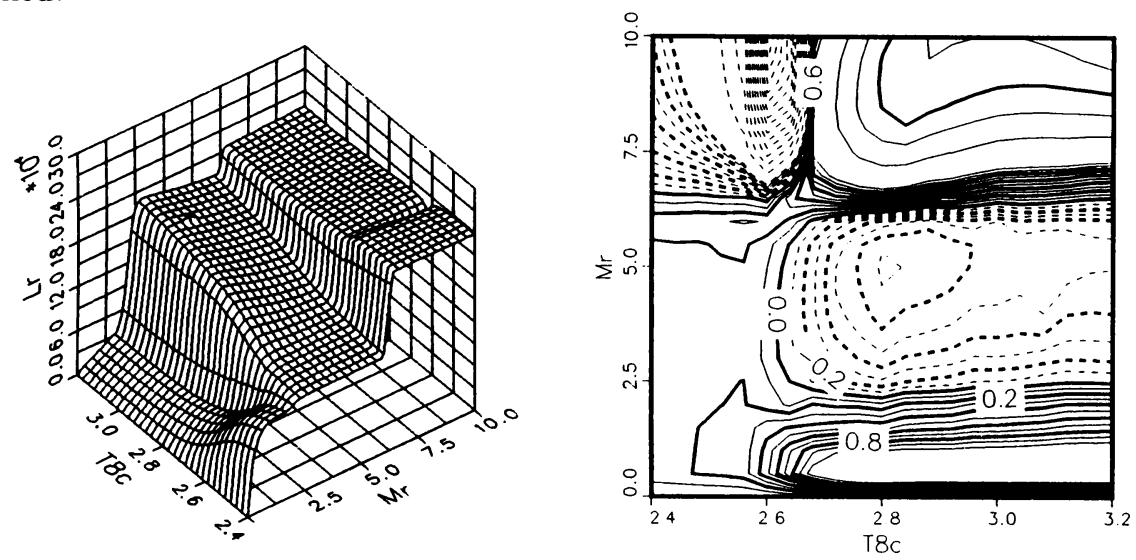

Fig. 3: a) Internal luminosity $L_{r} / L_{\odot}$ as function of the mass coordinate $M_{r} / M_{\odot}$ (spatial coordinate) and central temperature $T 8_{c}=T_{c} / 10^{8} \mathrm{~K}$ (time coordinate) at and beyond core He-exhaustion. The two steps indicate the locations of the He-burning (at $M_{r} \simeq 2 M_{\odot}$ ) and the H-burning (at $M_{r} \simeq 7 M_{\odot}$ ) shell sources. Note that the H-shell does not fade away when the He-shell source is activated. b) Contour plot of the inverse of the local timescale of density variation $d \ln \rho / d t$ for the same $M_{r}-T 8_{c}$ area as shown in Fig. 3a. Solid lines indicate contraction, dashed lines expansion. The units of $d \ln \rho / d t$ are on an arbitrary linear scale. For $T 8_{c} \gtrsim 2.6$ contracting core, expanding intermediate layers, and contracting envelope can be distinguished.

Note that a low envelope opacity seems also to be required in order to obtain the final envelope contraction, since calculations of a $20 M_{\odot}$ star and solar metallicity did not perform this contraction for any value of the semiconvection parameter $\alpha$. The reason for this metallicity dependence is not yet well understood and deserves future investigation.

Finally, it is important to say that alternative explanations for the (empirically undisputeable) blue-red-blue evolution of the SN 1987A progenitor have been proposed (cf. e.g. Arnett et al., 1989) and cannot be excluded. However, they all include assumptions about rotation or binarity, often in a somewhat arbitrary way. Furthermore, a track like that in Fig. 1 cannot only account for the properties of the SN 1987A progenitor, but also for many general properties of LMC supergiants, which is not discussed here due to space limitations.

\section{Formation of WR stars}

a) Stars above the Humphreys-Davidson limit

The absence of very luminous RSGs (Humphreys and Davidson, 1979) and the presence of WR stars can be recovered by stellar evolution calculations with standard input physics when a short phase with extremely high mass loss after core 
hydrogen exhaustion is included (Maeder, 1983). The coincidence, that a class of highly variable luminous stars - the LBVs - appears to have the right properties (i.e. HRD position, mean mass loss rate, number frequency, etc.; cf. Davidson et al., 1989) to fit to this short phase of high mass loss (now called LBV-phase) leads many astronomers to think that the LBV-scenario may in fact be the dominant formation channel for WR single stars. It has been shown that mass loss rate and duration of the high mass loss state needs not to be arbitrarily imposed in a stellar evolution calculation but can be obtained selfconsistently as result of the condition that a certain region of the HR diagram should be avoided by the evolutionary track (Langer, 1990). Typical mass loss rates and timescales obtained in this way are $\dot{M}_{L B V} \simeq 10^{-3} M_{\odot} y r^{-1}$ and $\tau_{L B V} \simeq 10^{4} y r$, but both numbers may vary significantly as function of the main sequence evolution (i.e. as function of the main sequence mass loss rate or assumptions on convection/semiconvection; see above). The product of both numbers, anyway, is constrained by the mass of the hydrogenrich envelope $\Delta M$ (i.e. the amount of mass with $X>X_{\text {crit }} ; X_{\text {crit }} \simeq 0.25$, cf. Langer and El Eid, 1986; Maeder and Meynet, 1987) left at the time of core H-exhaustion, i.e. $\dot{M}_{L B V} \cdot \tau_{L B V}=\Delta M$.

Note that still not much is known about the physical origin of the high LBV mass loss, which is not surprising in view of the diversity of observational features related with this very inhomogeneous class of stars (see contributions in: Davidson et al., 1989, and cf. Kiriakidis et al., these proceedings, for the case of $\eta$ Car).

\section{b) Stars below the Humphreys-Davidson limit}

The lower ZAMS mass limit for WR formation from single stars $M_{W R}$ is possibly lower than the critical mass limit which corresponds to the upper luminosity boundary for RSGs (i.e. $~ 10^{5.7} L_{\odot}$, cf. Humphreys and McElroy, 1984;
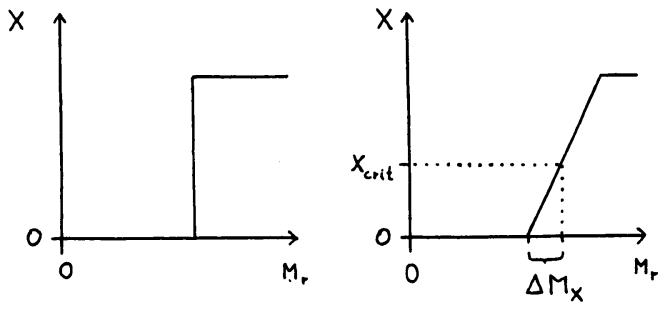

Fig. 4: Schematic H-profiles at time of core He-ignition.

$\left.\Rightarrow M_{Z A M S} \simeq 45 M_{\odot}\right)$ according to Schild and Maeder (1984), Humphreys et al. (1985), and van der Hucht et al. (1988). Consequently, a WR formation channel might exist, which involves a RSG stage. However, this cannot be concluded directly from stellar evolution calculations, basically due to the lack of theoretical predictions or strict observational constraints about $\mathrm{RSG}$ mass loss rates (cf. e.g. Jura, this volume). Also, it is not yet known whether an LBV-phase is associated with the RSG-channel of WR formation, either before or after the RSG stage. Note, however, that some LBVs (e.g. R71 or R110 in the LMC; cf. Wolf, 1989; Stahl et al., 1990) have luminosities well below $10^{5.7} L_{\odot}$, and some of their characteristics can be well understood when it is assumed that those objects are post-RSGs (Leitherer and Langer, 1990). Also Lortet (1989) concludes from an investigation of LBV environments that some of them might be post-RSGs (cf. also the contributions of R.M. Humphreys, C. Leitherer, and N. Walborn to this volume). 
Assuming that the evolutionary connection $\mathrm{RSG} \rightarrow(\mathrm{LBV}) \rightarrow \mathrm{WR}$ exists, then whether the subtype of the newly formed WR star is WNL or WNE (or better whether it is a hot or a cool WN star; see bolow) is simply a function of the internal hydrogen profile (cf. Langer, 1987, 1988). Fig. 4 is a schematic sketch of the two principle possibilities, i.e. a "steep" and a "flat" profile. Since all WR stars have small surface hydrogen concentrations (e.g. $X \lesssim 0.25$; cf. Hamann et al., these proceedings) only the part of the profile with $X \lesssim 0.25$ is relevant in this context. In case of a "steep" X-profile, the surface hydrogen mass fraction will change in a short time from a high abundance to zero, which means that the newly formed WR star will be hot (i.e. of type WNE). A "flat" X-profile gives rise to a cool WN phase of duration $\tau_{W N \text {-cool }} \simeq \Delta M_{X} / \dot{M}_{W N \text {-cool }}$.

Stellar evolution calculations indicate that the quantity $\Delta M_{X}$ (cf. Fig. 4) strongly increases with increasing ZAMS-mass. Though it is also a strong function of the incorporated physics (esp. again main sequence mass loss rate and convection/semiconvection models), it can be concluded that the WNL-phase (better the cool WN phase) lasts significantly longer for higher initial masses (cf. Langer, 1987; Maeder and Meynet, 1987; Maeder, 1990). Furthermore, when part of core He-burning is spent in the RSG regime before the WR formation phase, the $\mathrm{H}$ burning shell diminuishes $\Delta M_{X}$ due to transformation of $\mathrm{H}$ into He. This reduces additionally $\tau_{W N-\text { cool }}$ for post-RSG WR stars. Both effects together may account for the scarcity of cool WN stars with relatively low luminosities (Lundström and Stenholm, 1984; Conti, 1986)

\section{Structure and evolution of WR stars}

a) Effects of hydrogen

The presence of hydrogen in the envelope of a massive star has large consequences for its internal structure and thereby for its visual display and its evolution. Most important, the presence of hydrogen implies the presence of a hydrogen burning shell, due to which - finally as a consequence of the mirror principle; cf. Sect. $3 \mathrm{~b}$ - the star is much more extended and thus much cooler compared to hydrogenless stars. The larger extension is supported by the larger opacity and the smaller mean molecular weight of $\mathrm{H}$-containing matter compared to pure helium or metalrich mixtures. The radius $R_{*}$ (i.e. irrespective of the stellar wind) of H-containing WR stars is typically 10-20 times larger than the radius of WR stars of similar mass without hydrogen (cf. e.g. Langer and El Eid, 1986; Maeder and Meynet, 1987; Langer, 1990a). Note that this effect is nicely confirmed by the recent work of Hamann (this volume), who finds a clear correlation of the $\mathrm{H}$-abundance in WN stars with their effective temperature. A further consequence of the presence of hydrogen is a stabilization against vibrational pulsations (Maeder, 1985; Maeder and Schaller, this volume).

Because of the different internal structure and different physical and chemical surface composition of H-containing WR stars compared to WR stars without hydrogen, there is no reason to expect a common mass loss law for both types of WR stars, as discussed in Langer (1989b). Note, e.g., that the momentum problem for WR winds appears to be much smaller for cool WN stars as compared to H-less WR stars (cf. Langer, 1990).

Since the wind of WR stars may have a considerable optical thickness, the consequences of the presence of hydrogen for the apparent radius $R_{\text {eff }}$ (i.e. $R_{\text {eff }}:=$ $r(\tau=2 / 3))$ are not easy to predict. However, an order of magnitude estimate can 
be performed on the basis of Eqs. (8)-(15) of Langer (1989a), where it is shown that the optical thickness of the WR wind $\tau_{\text {wind }}=\tau\left(R_{*}\right)$ depends on mean opacity $\kappa$, mass loss rate and stellar radius as $\tau_{\text {wind }} \sim \kappa \dot{M} / R_{*}$. Order of magnitudes are $\tau_{\text {wind }} \simeq 1$ for cool (H-containing) WR stars, but $\tau_{\text {wind }} \simeq 10$ for WR stars without hydrogen, both for $\dot{M}=310^{-5} M_{\odot} y r^{-1}$.

b) Wolf-Rayet stars without hydrogen

WR stars without hydrogen - i.e. hot WNs, WCs, and WOs - have a very simple internal structure compared to H-containing WR stars: they are composed of a large convective He-burning core and a radiative envelope. Due to their high temperature, radiation pressure dominates over gas pressure within almost the whole star except a tiny surface layer. Due to this, and since the main opacity source is electron scattering, the structure of these objects is almost completely independent of their internal chemical composition, but is determined only by their actual mass and to a lesser extent - by their surface chemical composition (Langer, 1989a).

This finding has several important consequences. First of all, it means that the structure of H-less WR stars is independent of their previous evolution. This allows, e.g., to study those objects theoretically without considering how they have been formed. It explains the existence of a mass-luminosity relation for WR stars (Maeder, 1983) or a relation of the luminosity as a function of mass and surface composition, as well as similar relations for the radii or surface temperatures of WR stars (Langer, 1989a). It implies, e.g., that all H-less (hot) WN stars of the same metallicity should be located on a single line in the HR diagram, at least if rotaion or magnetic fields don't play any role.

A further consequence is - if again rotation and magnetic fields can be ignored; cf., anyway, Cassinelli (this volume) - that the mass loss rates of those objects should depend mainly on their actual mass, whatever its physical origin may be. Langer (1989b) investigated the evolutionary consequences of mass dependent WR mass loss and found a relation of the form

$$
\dot{M}_{W R}=(0.6-1.0) 10^{-7}\left(\frac{M_{W R}}{M_{\odot}}\right)^{2.5} / M_{\odot} y r^{-1}
$$

to yield the best agreement with many observed properties of galactic WR stars. Note that this agreement is rather insensitive to the actual power of the mass loss law, as long as it is $\gtrsim 1$. This result coincides with observational mass loss rates derived by Abbott et al. (1986) and is supported by the work of Smith and Maeder (1989).

One of the most prominent consequences of such mass dependent WR mass loss concerns the initial-final mass relation for massive stars (cf. Fig. 5): a) The final masses for stars which enter the WR phase during their evolution are very small, i.e. well below $10 M_{\odot}$, and b) The final masses of stars from a large interval of initial masses (say $\sim 35 M_{\odot}-\sim 100 M_{\odot}$ ) turn out to be the same within a very narrow limit $\sim \pm 1 M_{\odot}$; cf. Langer, 1989b). Note that for the highest initial masses (i.e. $M_{Z A M S} \gtrsim 100 M_{\odot}$ ), due to an increase of the duration of the H-containing WR phase with initial mass (cf. Sect. 3b), the possibility exists that some hydrogen is kept until the end of evolution. This would imply that those objects would not enter the regime of mass dependent mass loss, and they might remain very massive until the supernova phase (cf. Langer, 1987). The occurrence of this high mass 
final state depends on the mass loss rates of very luminous WN stars, for which no dependence on stellar parameter could yet be derived.

In a recent grid of stellar evolution sequences for a wide mass and metallicity range, calculated with mass dependent WR mass loss rates, Maeder (1990) confirmed the flat initial-final-mass relation of Fig. 5, and found also good agreement of the metallicity dependence of various WR subtype frequencies with observations (cf. Maeder, this volume).

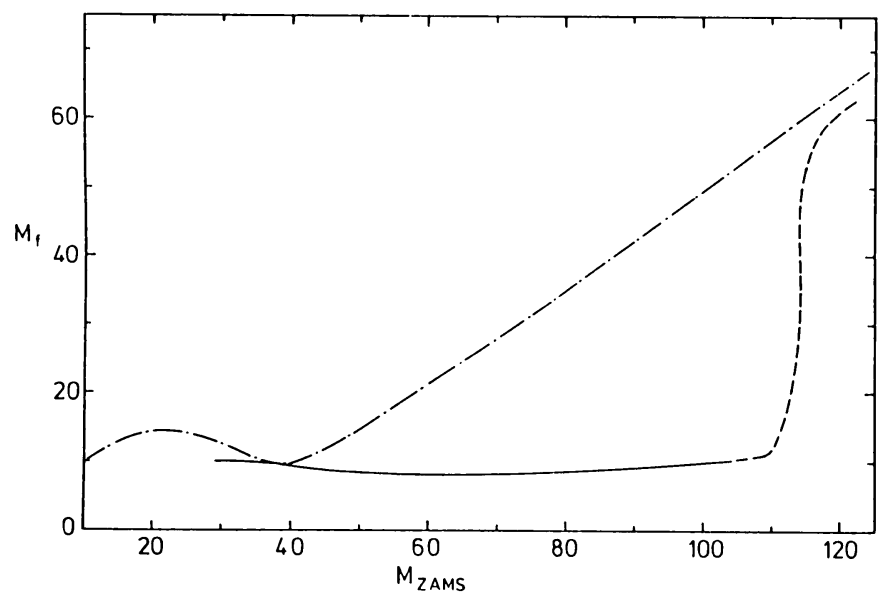

Fig. 5: Schematic initial-final-mass relation for massive stars in our Galaxy in the case where mass-dependent WR mass loss is taken into account (solid line), compared to previous models (dashed-dotted line; from Maeder and Meynet, 1987). The dashed part of the line corresponds to the possible existence of a very massive final state for the highest ZAMS-masses (see text).
Finally a comment on the "WN+WC" spectral type, which has been recently identified as being related to single WR stars rather than to WN+WC binaries (cf. Conti and Massey, 1989; Massey and Grove, 1989; Willis and Stickland, 1990). The implication is a partly mixed region between He-burning convective core and the layers above. In Langer (1990a) it is shown, that stellar evolution calculations

which include semiconvection (cf. Sect. $2 b$ ) yield a $\mathrm{WN}+\mathrm{WC}$ phase with a duration of some $10^{4} \mathrm{yr}$,

which agrees well with the observed $\mathrm{WN}+\mathrm{WC}$ frequency. Note that models which only use the Schwarzschild or the Ledoux criterion for convection or models including convective core overshooting do not obtain a $\mathrm{WN}+\mathrm{WC}$ phase. Therefore, the $\mathrm{WN}+\mathrm{WC}$ stars present a second argument in favour of semiconvection, which is completely independent of the SN 1987A progenitor evolution.

\section{Clues from supernovae for the evolution of massive stars}

Due to space limitations, this topic cannot be treated very explicit here. The reader is refered to Langer $(1990,1990 \mathrm{~b})$ for more details. Here, just the potential of supernova observations as tool for the analysis of the progenitor evolution is briefly summarized.

- Light curves and spectra of Type II SNe allow estimates of envelope mass and He-core mass of the progenitor, and thus an estimate of the amount of mass lost during the whole hydrostatic evolution (cf. e.g. Woosley, 1988). The inhomogeneity of the Type II SN class (cf. Nomoto, this volume) may reflect the 
diversity of possible H-containing pre-SN configurations (WN, BSG, RSG; all with different envelope masses).

- WR stars of any subtype may be pre-SN configurations. However, the bulk of galactic WR stars $\left(40 M_{\odot} \lesssim M_{Z A M S} \lesssim 100 M_{\odot}\right)$ is supposed to end evolution as low mass WC/WO star (Langer, 1989b, Maeder, 1990).

- A relation of the final low mass WC/WO stage to Type Ib and/or Ic SNe (cf. contributions of A. Fillipenko and K. Nomoto to this volume) cannot be excluded on the basis of the light curve alone (cf. Langer and Woosley, this volume). A confirmation would strongly support the concept of mass dependent WR mass loss.

- The existence of a final high mass WR stage $\left(\gtrsim 60 M_{\odot}\right)$ could in principle be confirmed by observations of the characteristics of an $\mathrm{e}^{ \pm}$-pair creation supernove (cf. El Eid and Langer, 1986; Herzig et al., 1990).

Clearly, much progress for the understanding of massive star evolution has to be expected from the field of SN studies in the near future.

Acknowledgement. The author is grateful to Prof. B. Hidayat and his colleagues at Bosscha Observatory for their warm hospitality. Travel support by the Deutscher Akademischer Austauschdienst (DAAD) and the Deutsche Forschungsgemeinschaft (DFG) is also gratefully acknowledged. This work has been supported in part by DFG grants La 587/1-2 and Fr 325/28-2.

\section{References}

Abbott, D.C.: 1982, Astrophys. J. 259, 282

Abbott, D.C.,Bieging, J.H., Churchwell, E., Torres, A.V.: 1986, Astrophys. J. 303, 239

Arnett, W.D., Bahcall, J.N., Kirshner, R.P., Woosley, S.E.: 1989, Ann. Rev. Astron. Astrophys. 27, 629

Castor, J.I., Abbott, D.C., Klein, R.I.: 1975, Astrophys. J. 195, 157

Chiosi, C., Summa, C.: 1970, Astrophys. Space Sci. 8, 478

Chiosi, C., Nasi, E.: 1978, Astrophys. Space Sci. 56, 431

Chiosi, C., Maeder, A.: 1986, Ann. Rev. Astron. Astrophys. 24, 329

Conti, P.S.: 1986, IAU-Sympos. 116, 199

Conti, P.S., Massey, P.: 1989, Astrophys. J. 337, 251

Davidson, K., Moffat, A.F.J., Lamers, H.J.G.L.M.: 1989, Physics of Luminous Blue Variables, Proc. IAU-Coloqu. 113, Kluwer

Eggleton, P.P.: 1972, Mon. Not. Royal Astron. Soc. 156, 361

El Eid, M.F., Langer, N.: 1986, Astron. Astrophys. 167, 274

Fricke, K.J., Strittmatter, P.A.: 1972, Mon. Not. Royal Astron. Soc. 156, 129

Herzig, K., El Eid, M.F., Fricke, K.J., Langer, N.: 1990, Astron. Astrophys. , in press

van der Hucht, K.A., Hidayat, B., Admiranto, A.G., Supelli, K.R., Doom, C.: 1988, Astron. Astrophys. 199, 217

Humphreys, R.M., Davidson, K.: 1979, Astrophys. J. 232, 409

Humphreys, R.M., McElroy, D.B.: 1984, Astrophys. J. 284, 565

Humphreys, R.M., Nichols, M., Massey, P.: 1985, Astron. J. 90, 101

Iben, I., Jr.: 1974, Ann. Rev. Astron. Astrophys. 12, 215

Kato, S.: 1966, P.A.S.J. 18, 374

Kippenhahn, R., Weigert, A.: 1990, Stellar Structure and Evolution, Springer 
Kozlowski, M.: 1971, Astrophys. Letters 9, 65

Kudritzki, R.P., Pauldrach, A., Puls, J., Abbott, D.C.: 1989, Astron. Astrophys. 219, 205

Langer, N.: 1986, Astron. Astrophys. 164, 65

Langer, N.: 1987, Astron. Astrophys. 171, L1

Langer, N.: 1988, in: Proc. IAU-Colloq. 113, p. 221

Langer, N.: 1989a, Astron. Astrophys. 210, 93

Langer, N.: 1989b, Astron. Astrophys. 220, 135

Langer, N.: 1990, in: Angular Momentum and Mass Loss for Hot Stars, proc. $2^{\text {nd }}$ Ames-Trieste Workshop, L.A. Wilson, R. Stalio, eds., in press

Langer, N.: 1990a, in: Properties of Hot Luminous Stars, proc. $1^{\text {st }}$ Boulder-Munich Workshop, C. Garmany, ed., A.S.P. Conf. Ser. Vol. 7, p. 328

Langer, N.: 1990b, in: Supernovae, proc. $10^{\text {th }}$ Santa-Cruz Summer Workshop, S.E. Woosley, ed., in press

Langer, N., Sugimoto, D., Fricke, K.J.: 1983, Astron. Astrophys. 126, 207

Langer, N., El Eid, M.F., Fricke, K.J.: 1985, Astron. Astrophys. 145, 179

Langer, N., El Eid, M.F.: 1986, Astron. Astrophys. 167, 265

Langer, N., El Eid, M.F., Baraffe, I.: 1989, Astron. Astrophys. 224, L17

Langer, N., El Eid, M.F., Baraffe, I.: 1990, in: Supernovae, proc. $10^{\text {th }}$ Santa-Cruz Summer Workshop, S.E. Woosley, ed., in press

Langer, N., El Eid, M.F.: 1990, in preparation

Lauterborn, D., Refsdal, S., Weigert, A.: 1971a, Astron. Astrophys. 10, 97

Lauterborn, D., Refsdal, S., Weigert, A.: 1971b, Astron. Astrophys. 13, 119

Ledoux, P.: 1941, Astrophys. J. 94, 537

Leitherer, C., Langer, N.: 1990, in: IAU-Symp. 148, in press

de Loore, C.: 1980, Space Sci. Rev 26, 113

Lortet, M.C.: 1989, in: Proc. IAU-Colloq. 113, p. 45

Lundström, I., Stenholm, B.: 1984, Astron. Astrophys. Suppl. 58, 163

Maeder, A.: 1983, Astron. Astrophys. 120, 113

Maeder, A.: 1985, Astron. Astrophys. 147, 300

Maeder, A.: 1990, Astron. Astrophys. Suppl. 84, 139

Maeder, A., Meynet, G.: 1987, Astron. Astrophys. 182, 243

Massey, P., Grove, K.: 1989, Astrophys. J. 344, 870

Uwocki, S.P., Castor, J.I., Rybicki, G.B.: 1988, Astrophys. J. 335, 914

Pauldrach, A., Puls, J., Kudritzki, R.P.: 1986, Astron. Astrophys. 164, 86

Pauldrach, A., Kudritzki, R.P., Puls, J., Butler, K.: 1990, Astron. Astrophys. 228, 125

Renzini, A.: 1987, Astron. Astrophys. 188, 49

Schild, H., Maeder, A.: 1984 Astron. Astrophys. 136, 237

Smith, L.F., Maeder, A.: 1989, Astron. Astrophys. 211, 71

Stahl, O., Wolf, B., Klare, G., Juettner, A., Cassatella, A.: 1990, Astron. Astrophys. 228, 379

Stothers, R., Chin, C.-W.: 1976, Astrophys. J. 204, 472

Weaver, T.A., Zimmerman, G.B., Woosley, S.E.: 1978, Astrophys. J. 225, 1021

Willis, A.J., Stickland, D.J.: 1990, in: Properties of Hot Luminous Stars, proc. $1^{\text {st }}$ Boulder-Munich Workshop, C. Garmany, ed., A.S.P. Conf. Ser. Vol. 7, p. 354

Wolf, B.: 1989, in: Proc. IAU-Colloq. 113, p. 91

Woolsey, S.E.: 1988, Astrophys. J. 330, 218 


\section{DISCUSSION}

Niemela: If the progenitor of SN1987A in the LMC had $20 M_{\odot}$, would that preclude smaller mass progenitors for WR stars? In other words: the initial mass for WR stars should be $>20 M_{\odot}$.

Langer: For single stars, yes. Note, however, that due to the metallicity dependence in stellar evolution you cannot transfer this limit to, e.g., the Milky Way.

Moffat: I agree that $\dot{M} \sim M^{\alpha}$ with $\alpha$ positive, but observations (e.g., from polarization of WR $+O$ binaries which yield $M$ and $\dot{M}$ suggest that $\alpha \approx 1.5$ not 2.5 .

Langer: I can live as well with $\alpha \approx 1.5$. My results indicate that the exact value of $\alpha$ is not important. The main point I wanted to make is that it should be clearly $\alpha>0$, note that in the past $\alpha=0$ has been used almost exclusively.

Schulte-Ladbeck: I found your illustration of stellar interiors using different criteria very illuminating. I have two questions. The first concerns rotational mixing and goes to you and Maeder: do we now have a number of evolutionary tracks with rotation? The second one is about semi-convection and the smaller cores (e.g., what we need in binaries) and should perhaps be addressed to our binary theorist, De Greve. What is our current standing on binary models with semi-convection (and with conservative/non-conservative Roche-lobe overflow)?

Langer: To your first point: I recently calculated a SN1987A progenitor track including the rotational induced baroclinic instability, which was found to account for the high observed $N$-enrichment. Also, e.g., Maeder and Sreenivasan calculated massive star track including rotational effects. However, it is my opinion that prescription of the involved rotational physics is still to a large degree arbitrary and/or uncertain. For your second point: I do not know any binary evolution calculation including the effect of semi-convection as I understand it (e.g., as a physical instability).

Vanbeveren: I was very pleased with your model for SN1987A where you were able to explain the progenitor without core overshooting. The OBN binary HD 163181 consists of a $13 M_{\odot}(\mathrm{OBN})$ component and a $22 M_{\odot}$ companion. Since the OBN star is $\pm 1.5 \mathrm{mag}$ brighter than the $22 M_{\odot}$ star, it should be a core helium burning star (after a possible Roch-lobe overflow). But, why then is it not a WR star? A possible solution can be found when non-overshooting models are used and let semi-convection play an important role.

Langer: I would appreciate that binary evolutionists would take semi-convection into account. However, note that in binaries semi-convection is already important during central $H$-burning for the mass gainer, since its $H$-burning convective core will tend to grow, leading to a composition discontinuity at its top.

Yungelson: You do predict very low masses of pre-SN. This means that a low amount of mass will be ejected at SN explosions. Would this not lead to overproduction of binary pulsars?

Langer: The low mass of the pre-supernova star does not automatically mean that you create a pulsar. Since the whole star is a metal-rich object and since it "remembers" its high mass origin, you may possibly create a black hole rather than a neutron star.

Barlow: You mentioned that LBV's should become WNE's if there is a sharp composition boundary between hydrogen and helium, while they should become WNL's if there is a smooth composition gradient. The results that I presented on Tuesday on the LBV's P Cyg and AG Car showed that their winds have intermediate compositions with very enhanced $\mathrm{He} / \mathrm{H}$ ratios. This would seem to argue in favour of there being a smooth composition gradient in the envelopes of these stars. 
Langer: Partly this is true. However, they could still transform directly into WNE's, if the hydrogen gradient in the remaining envelope is very steep.

Massey: The designation WNL and WNE has to do with the relative strength of $N V$ and $N I I I$, and is not based on $\mathrm{H} / \mathrm{He}$ ratios. HD 177230 , a WN 8 "spectrum standard" (according to Beals!), has no $H$ - even Underhill agreed with that in 1981 . One of the three known galactic WN 3 stars does show $H$. I just think the situation is more complicated than implied by using two shoe boxes, and assuming that everything in each box is the same.

Langer: I am sorry if I created confusion by my use of the designations WNL and WNE for hydrogen containing and hydrogen less WN stars. I am aware that the correlation of the hydrogen abundance with WN spectral subtype is not unambiguous, as also demonstrated by Hamann at this symposium.

Nomoto: (1) Regarding the evolution of the SN1987A progenitor, helium enhancement observed in the circumstellar shell would require an additional mixing process in your model. (2) In your model of the SN1987A progenitor, what is the effect of low metallicity for the blueward evolution? (3) How much is the critical value of metallicity that divides the BSG and RSG progenitor?

Langer: (1) If the high helium abundance for the progenitor of SN1987A is confirmed, it poses a problem to our model. (2) Higher $Z$ implies higher opacities which favours extended envelopes. (3) We have done calculations only for $Z=0.5 \%$ (LMC) and $Z=2 \%$ (Milky Way). For the latter case we obtained RSG pre-SN configurations independent of the semi-convection parameters.

Maeder: I think it is fair to say that several explanations of the blue progenitor of SN1987A have been proposed. Among them, the explanation by Nomoto and coworkers looks very attractive and my models also will support it. During the $C$-burning phase, the external and intermediate convective zones come so close to each other, that any extension of convection would mix the materials from both zones. This results into a blue location in the HRD due to the increased opacity and also this explains the $\mathrm{He}$ and $\mathrm{N} / \mathrm{C}$ observed enhancements. Your criterion for semi-convection explains the blue location of the SN progenitor, but not the $\mathrm{He}$ and $\mathrm{N} / \mathrm{C}$ enhancements. I think this should be properly stated for the clarity of the debate about the $\mathrm{SN}$ progenitor.

Langer: Since this is a symposium on WR stars, I tried to concentrate on these objects in my talk and did not take too much time for the discussion of the SN1987A progenitor. Otherwise, I might have been able to convince you that the semi-convection explanation of the pre-SN evolution as well explains many general properties of LMC supergiant stars. Also the high observed $N$-enrichment in the SN progenitor can be easily accounted for due to the baroclinic instability put forward by yourself a few years ago. A detailed paper about this topic is in preparation. Concerning the SN progenitor model of Nomoto et al., I just am a bit worried, since both the amount of mixing invoked and the time of mixing are not yet physically justified.

Sreenivasan: I think one can produce an acceptable evolutionary pattern of the progenitor of SN 1987A without any tricks about the lines outlined in my poster (details in the references). Your method depends upon the way you treat semi-convection! But one cannot self-consistently use the Ledoux gradient without an independent equation to determine the $\mu$-gradient. Once you mix, you alter the $\mu$-gradient according to your mixing scheme and not according to the physics of the interior. That is why using the Ledoux criterion is inconsistent. Finally, the semi-convection you see in the $\mathrm{He}$-burning stage is different from what one sees in the $\boldsymbol{H}$-burning stage and the kind discussed for horizontal branch stars by Giannone et al. (1971). The two arise for quite different reasons and your diffusion 
coefficient with its $\alpha$ factor is also very curious. It is not very clear what really goes on in your model!

Langer: There is much confusion about what semi-convection means in the literature. The only way I can understand semi-convection is as a physical instability, as discussed by Kato (1966). Especially, the physical origin of the semi-convection in $H$ - and $H e$-burning phases is the same in our models. Furthermore, we do not encounter the numerical problems or inconsistencies you mention. Our method is comprehensively described in Langer et al. (1985).

Leitherer: Stars evolve to the red in the HRD, and - depending on how much hydrogen-rich material you remove from the surface - they may evolve back to the blue part of the HRD. What is the actual physical reason for this behaviour?

Langer: There are two possibilities for a star to return to hotter surface temperatures in the HR diagram: (1) so called blue loops and (2) transition to the WR phase, and I presume you refer to the second case. There, I think, the physical reason is twofold. You increase the average mean molecular weight in the envelope (which becomes more $\mathrm{He}$-rich) and reduce the opacity; both tend to decrease the stellar radius. Additionally, the temperature in the $H$-burning shell is reduced as less and less mass lies above it. Since a shell source increases the total stellar radius appreciably ( $c f$. Cox \& Giuli), its extinction means that the radius shrinks, i.e., the surface temperature increases.

Norbert Langer

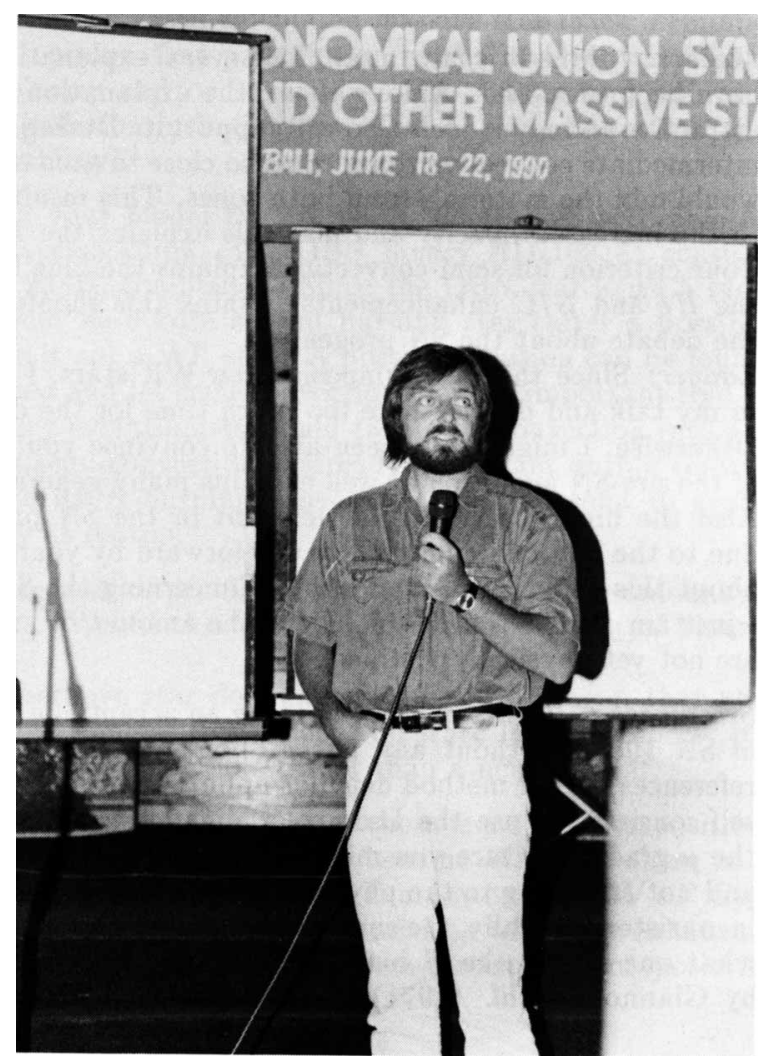

